\title{
Measles outbreak in Greater Manchester, England, October 2012 to September 2013: epidemiology and
} control

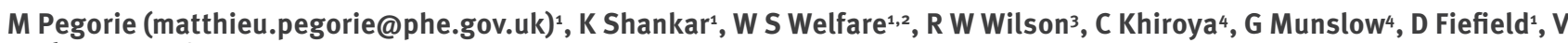

Bothra ${ }^{1}, \mathbf{R}$ McCann ${ }^{1,2}$

1. Health Protection Team, Greater Manchester Public Health England Centre, Public Health England, Manchester, United Kingdom

2. Manchester Academic Health Sciences Centre, University of Manchester, Manchester, United Kingdom

3. Manchester Medical School, University of Manchester, Manchester, United Kingdom

4. Greater Manchester Screening and Immunisation Team, NHS England/ Public Health England, Manchester, United Kingdom

Citation style for this article:

Pegorie M, Shankar K, Welfare WS, Wilson RW, Khiroya C, Munslow G, Fiefield D, Bothra V, McCann R. Measles outbreak in Greater Manchester, England, October 2012 to September 2013: epidemiology and control. Euro Surveill. 2014;19(49):pii=20982. Available online: http://www.eurosurveillance.org/ViewArticle. aspx?Articleld $=20982$

Article submitted on 18 March 2014 / published on 11 December 2014

This paper describes the epidemiology and management of a prolonged outbreak of measles across the 2.7 million conurbation of Greater Manchester in the United Kingdom. Over a period of one year (from October 2012 to September 2013), over a thousand suspected measles cases $(n=1,073)$ were notified across Greater Manchester; of these, 395 (37\%) were laboratory-confirmed, 91 (8\%) were classed as probable, 312 (29\%) were classed as possible and 275 (26\%) excluded. Most confirmed and probable cases occurred in children within two age groups - infants (too young to be eligible for measles-mumps-rubella (MMR) vaccination according to the national immunisation programme) and children aged 10-19 years (low vaccine uptake in this cohort because of unfounded alleged links between the MMR vaccine and autism). During this one year period, there were a series of local outbreaks and many of these occurred within the secondary school setting. A series of public health measures were taken to control this prolonged outbreak: setting up incident management teams to control local outbreaks, a concerted immunisation catch-up campaign (initially local then national) to reduce the pool of children partially or totally unprotected against measles, and the exclusion of close contacts from nurseries and school settings for a period of 10 days following the last exposure to a case of measles.

\section{Background}

The number of notified measles cases in Europe fell from 28,203 to 7,499 between 2003 and 2009 [1]. Since then, there have been a number of measles outbreaks especially in central and western Europe, with a peak of cases reported in $2011(32,124$ cases reported) [1,2]. France was the most affected country, with $47 \%$ of cases in Europe in 2011, while several other countries have also reported a considerable number of cases including Bulgaria, Germany, Italy, Romania, Spain, Ukraine, and the United Kingdom (UK) $[1,2]$.

In the UK, a measles-containing vaccine was introduced into the national childhood immunisation schedule in 1968. Since 1988 , the combined measlesmumps-rubella (MMR) vaccine has been used [3]. In the early 1990s, the number of confirmed cases of measles fell significantly from ca 80,000 cases in 1988 to ca 100 cases in 2000 , as uptake of MMR vaccination increased, to the point where sustained transmission was interrupted [4]. Unfounded fears about a potential link between MMR vaccine and autism $[5,6]$ in the late 1990s damaged public confidence in MMR vaccine, reduced uptake, and increased the risk of sustained transmission $[7,8]$ leading to the number of confirmed measles cases in England and Wales increasing to above 1,000 in 2008 and remaining above 1,000 since, except for the year 2010 when 380 cases were confirmed. In the UK, recent outbreaks have mainly occurred in areas or specific groups with known low coverage of MMR vaccination [9].

Greater Manchester is a city region in the north-west of England with a population of around 2.7 million, administratively divided into 10 local authorities with different population sizes and characteristics. Recent MMR vaccine uptake has been high, with $93.3 \%$ of children having received their first dose of MMR vaccine by their second birthday in 2011-2012 (national mean: $91.2 \%$ ) and $88.1 \%$ of children having received two doses of MMR vaccine by their fifth birthday in 20112012 (national mean: 86\%) [10].

However, there was an under-vaccinated cohort aged between 10 and 16 years across Greater Manchester, estimated at 31,600 children in April 2013. Of these, 11,993 were totally unvaccinated, and $19,644 \mathrm{had}$ 
Number of notifications of suspected and confirmed cases of measles, Greater Manchester, England, 2007-2013

\begin{tabular}{|l|c|c|}
\hline Year of receipt of notifications & Total number of notifications received & $\begin{array}{c}\text { Number of laboratory-confirmed measles cases } \\
\text { among the notifications received }\end{array}$ \\
\hline 2007 & 212 & 22 \\
\hline 2008 & 235 & 26 \\
\hline 2009 & 394 & 40 \\
\hline 2010 & 310 & 30 \\
\hline 2011 & 246 & 21 \\
\hline 2012 & 664 & 229 \\
\hline 2013 & 894 & 149 \\
\hline
\end{tabular}

received only one dose of MMR vaccine [11]. Of those aged 10-16 years in Greater Manchester, $10.4 \%$ were thought to be susceptible to measles [12]. This cohort has been the target of previous local catch-up vaccination campaigns with varying success [12] and was being targeted by a national catch-up vaccination campaign (2013-2014) [13].

\section{Surveillance system in England}

All doctors in England and Wales must notify the 'Proper Officer' of the relevant local authority (usually staff of the local Public Health England Centre) of clinically-suspected cases of measles. Other clinicians are encouraged to do the same.

The local Public Health England (PHE) team will record and undertake local follow-up including assessing how likely this is to be measles. The local team will send an oral fluid swab kit to the suspected case. This kit is designed for use by the patient and is posted directly to the Virus Reference Department, PHE Microbiology Services Colindale, London (the national reference laboratory) for IgM / IgG and RNA testing.

Test results are sent to both the relevant clinician and PHE. All laboratories in England notify PHE of confirmed measles cases. PHE receives and collates reported cases with a negative result and positive cases and reports confirmed cases to the European surveillance network (EUVAC-NET) on a monthly basis. This is described in more detail elsewhere [14].

In this report, we describe a prolonged outbreak of measles in Greater Manchester, the control measures that were taken and the lessons learned.

\section{Methods}

This report includes all reported cases of measles in residents of Greater Manchester between 1 October 2012 and 30 September 2013.

\section{Case definitions}

Case definitions were primarily devised for case management purposes and were adapted from national guidelines [3] to fit the local epidemiology of the outbreak. The national case management guidelines separate likely (probable) cases and unlikely (possible) cases. These are consistent with those used in a recently published outbreak report [15].

\section{FIGURE 1}

Number of confirmed, probable and possible cases of measles by week of rash onset, Greater Manchester, England, October 2012-September $2013(\mathrm{n}=798)$

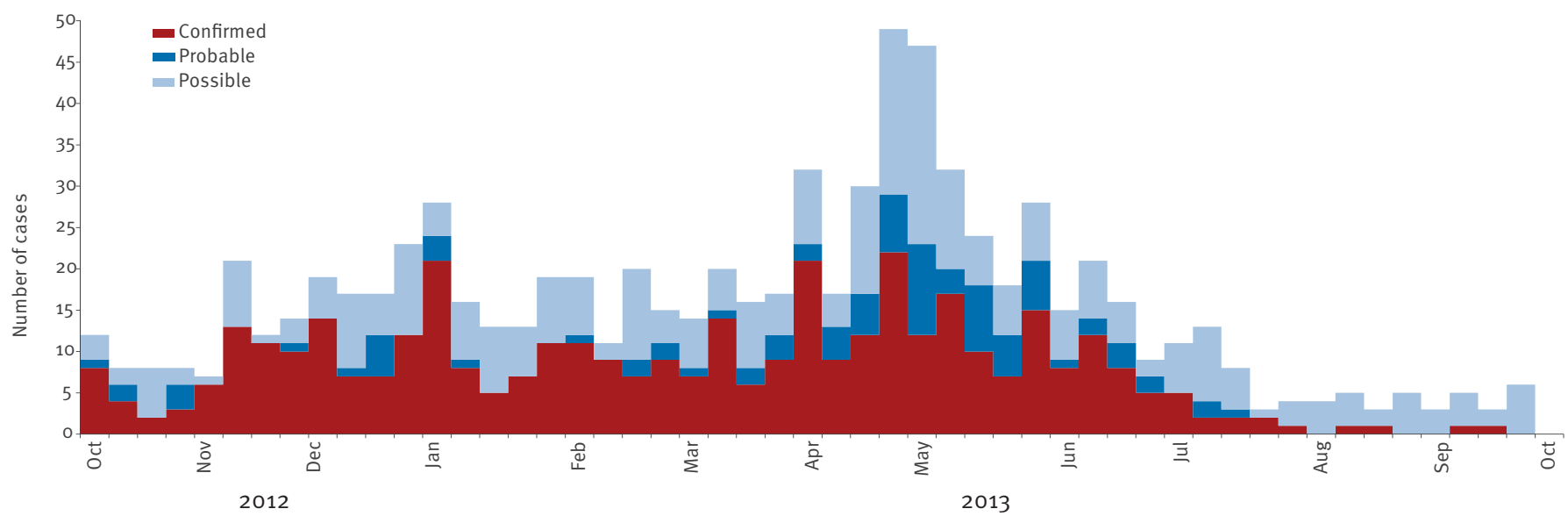


Measles rates by Middle Super Output Area (MSOAs)a , Greater Manchester, England, October 2012-September 2013 (n=486 probable and confirmed cases)

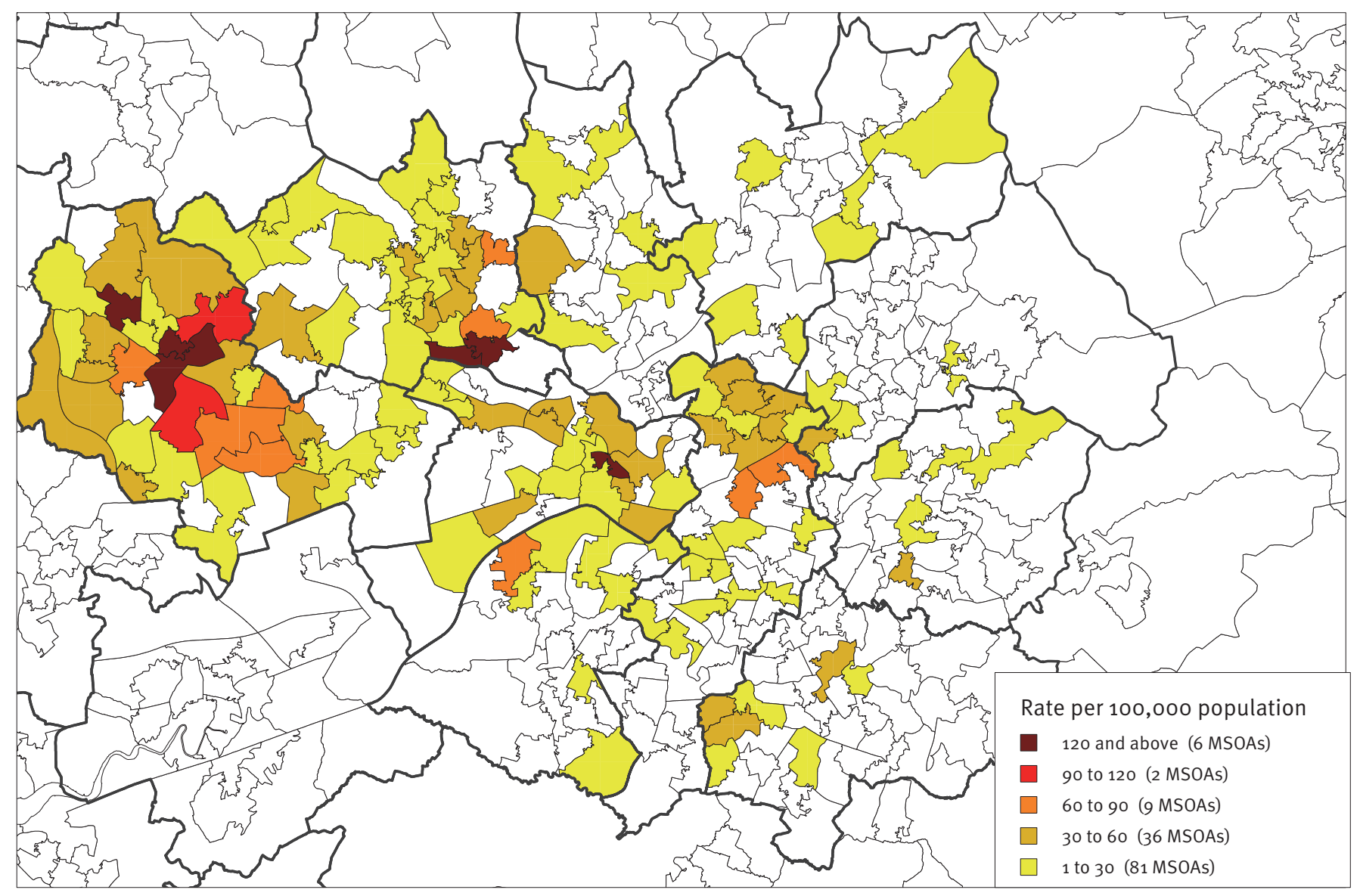

Super Output Areas (SOAs) are small areas of consistent size across the country used by the United Kingdom Office for National Statistics and are not subjected to regular boundary change. Each Middle Super Output Area (MSOA) has a population of 5,000-15,000 people and contains 2,000-6,000 households.

Confirmed case: measles IgM-positive result in blood or oral fluid in the absence of a history of recent vaccination or confirmed wild measles RNA positive in any clinical specimen.

Probable case: case notified by a clinician as suspected measles who was not fully vaccinated with two doses of MMR vaccine and met one or more of the following criteria: (i) epidemiological link (recent contact with a laboratory-confirmed case of measles); (ii) member of the travelling community or orthodox Jewish community; (iii) link to a setting with a known outbreak; or (iv) aged over 16 . Such cases are classified as likely as per national guidelines. If a laboratory result was received for a probable case, they were reclassified as confirmed or discarded depending on the result.

Possible case: case notified by a clinician as suspected measles but not meeting the above criteria (classified as unlikely as per national guidelines [1]). If a laboratory result was received for a possible case, they were reclassified as confirmed or discarded depending on the result.
Discarded case: a previously notified case with negative test result at the national reference laboratory in an adequate and appropriately timed specimen of oral fluid or blood.

Cases notified who were from the orthodox Jewish community and the travelling community were considered to be more likely to be actual measles based on known low vaccine coverage in these groups, increased risk of introduction of measles and recent local epidemiology. Confirmed, probable and where appropriate, possible cases were included in this analysis whereas discarded cases were excluded. To ensure that this report describes a single outbreak, cases (and associated contacts) with confirmed genotype other than D8 were excluded. Only one case with a genotype other than D8 was identified during the period reported here: a case with genotype $B 3$.

Reporting clinicians were asked to submit samples for local PCR by the PHE laboratory in Manchester, where possible. A salivary testing kit was posted to all reported cases for self-administration and direct return to the national reference laboratory. 
The likely site of measles transmission or acquisition associated with each case was recorded. If two or more cases were linked to a particular setting, further investigation was undertaken, including social network analysis. Social network diagrams were drawn using Microsoft Visio.

\section{Strategic coordination}

Outbreak control teams (OCT) were established for affected individual local authorities, with oversight by a Measles Strategy Group. The strategy group reviewed case definitions based on the evolving epidemiology of the outbreak and agreed on the strategy for optimising efficiency of the overall outbreak response. In specific geographical areas, for specific periods of time where community transmission of measles was considered endemic, MMR vaccination was prioritised over the public health management of cases and contacts.

Proactive and reactive communication strategies were agreed by the strategy group. Examples include proactive engagement with local media through press releases and interviews, and regular updates which were communicated to local authorities affected by the outbreak and to clinical networks throughout Greater Manchester.

\section{Results}

Outbreak description

Between 1 October 2012 and 30 September 2013, 1,073 suspected cases of measles in Greater Manchester residents were reported by clinicians and laboratories (Figure 1). This was significantly higher than expected (Table). Of these cases, 670 were tested. Of all suspected cases reported by clinicians and laboratories, 395 (37\%) were confirmed, 91 (8\%) were classed as probable, 312 (29\%) were classed as possible and 275 (26\%) were discarded after laboratory results were known.

The highest number of reports in one week was in April 2013 (Figure 1). The outbreak mainly affected four of ten Greater Manchester local authority areas: Bolton and Wigan initially, then Salford, and lastly, Manchester (Figure 2). In 2013, there was a sharp decrease in the number of cases notified, following the start of the summer school holidays (from the end of July until early September) (Figure 1).

Two peaks were seen in the age distribution (Figure 3): those aged under two years and those aged 10-14 years. If possible cases were included (data not shown) the under two-year-olds peak would be more pronounced, probably as younger children are more

\section{FIGURE 3}

Measles-mumps-rubella vaccination status by age of confirmed and probable measles cases, Greater Manchester, England, October 2012-September 2013

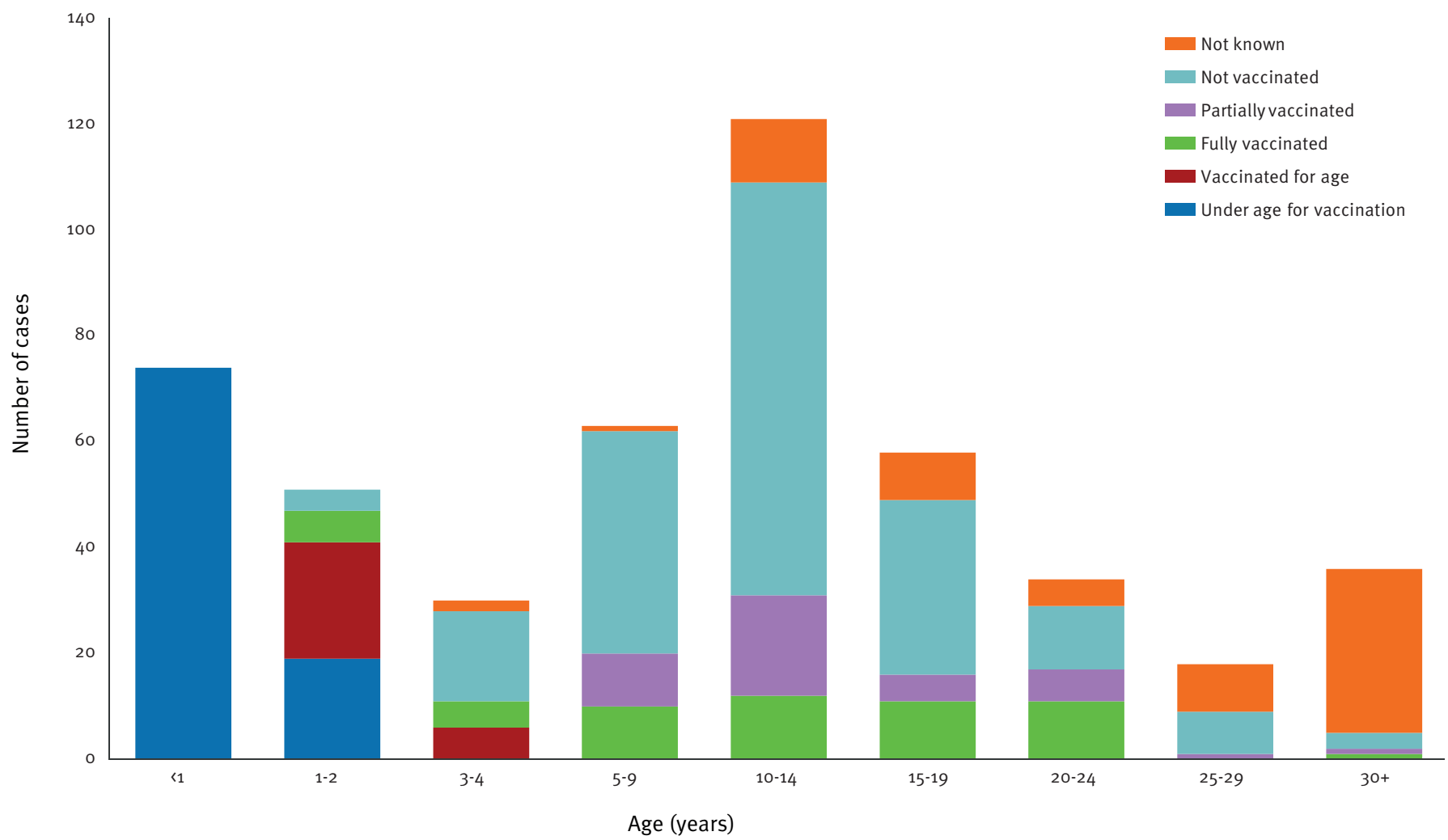


Example of a social network diagram representing transmission events in a high school and in household settings

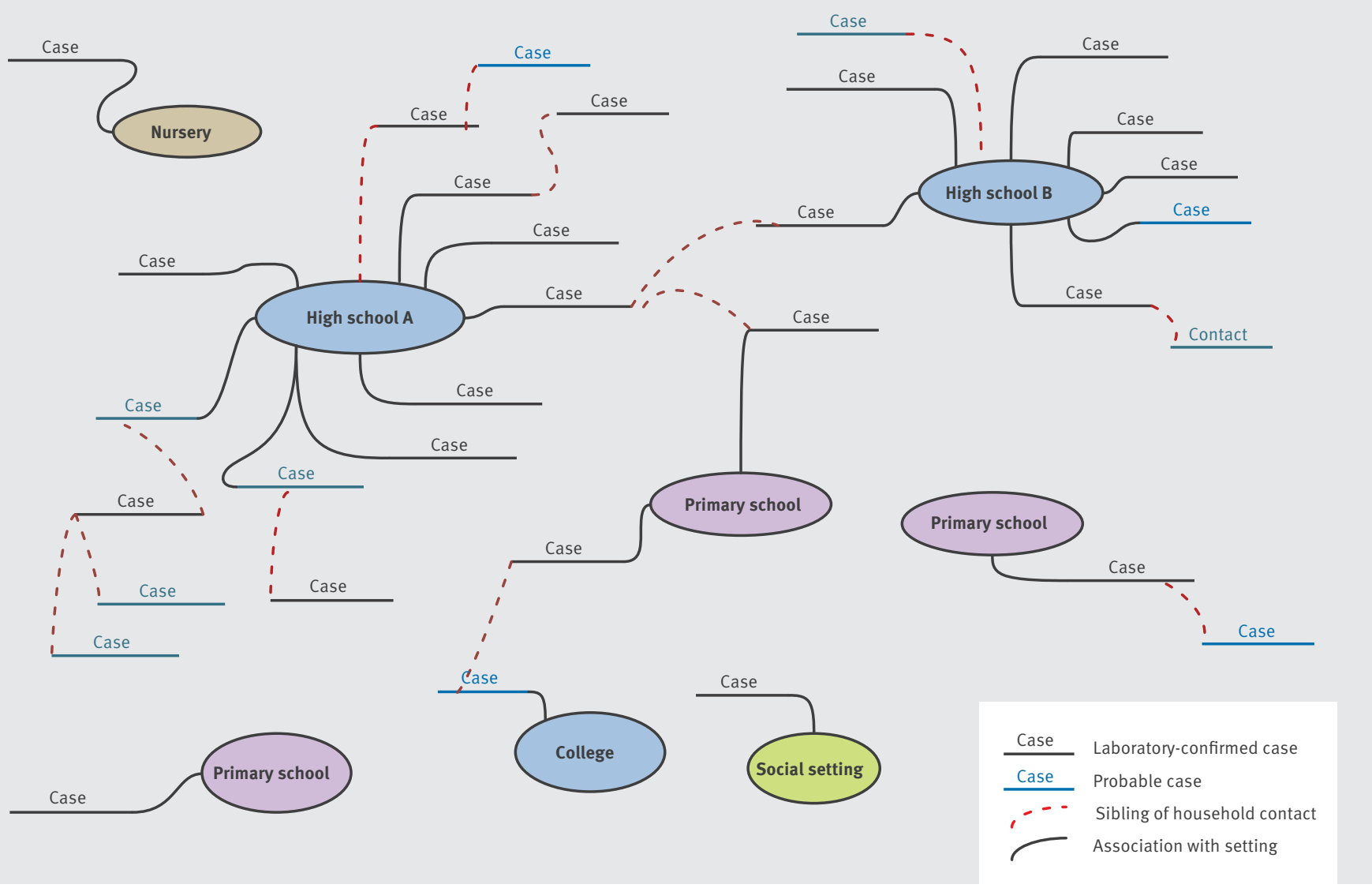

likely to present to health services with rash illnesses. Information on age was available for all possible, probable and confirmed cases. The median age among confirmed cases alone was 21 years (range 0-52 years), and among confirmed and probable cases, 23 years (range 0 to 52 years). Among probable and confirmed cases $54 \%$ (264) were male. The majority of probable and confirmed cases were in those who had not had two doses of MMR vaccine. Of 486 probable and confirmed cases, only 56 cases ( $12 \%$ ) were fully vaccinated with two doses of MMR vaccine (Figure 3).

Among the 486 confirmed and probable cases, 69 $(14 \%)$ cases did not know if they were vaccinated ('Not known' in Figure 3). Of these, almost 50\% were above 30 years of age.

\section{Schools and social networks}

More than 40 educational settings were involved in this outbreak, mainly secondary schools. Eight schools had more than five cases each. The two schools with the highest number of cases (ca20) were both large secondary schools (over 1,000 pupils) with similar numbers of children who had received one dose or no dose of MMR vaccine in each school.
Due to the high proportion of susceptible 10-19 yearolds in secondary schools and the high levels of social interaction within secondary schools, a number of secondary schools were identified as probable sites of transmission. On average, within the same school, $56 \%$ (95\% confidence interval $(\mathrm{Cl}): 44-67)$ of secondary cases were in the same year group as the index case, and this proportion increased with index case age, suggesting that compared with younger school year groups, in the older school year groups, transmission events were more likely to occur between children from the same school year group (unpublished data).

Members of the Measles Strategy Group reviewed periodically the confirmed and probable cases along with any associated educational or community settings, and produced the network diagram. This network diagram was found useful to the team handling the outbreak to get a quick view of the spread of the disease.

\section{Laboratory results}

In total, 670 of the cases linked to this outbreak were tested. Genotype D8 was the predominant genotype identified in cases.

Data on hospitalisations due to secondary complications were not of adequate quality to enable analysis. 


\section{Control measures}

It was not feasible or possible to formally evaluate the local control measures implemented due to resource limitations and associated opportunity cost to the health protection team, and the challenges of finding an appropriate design for evaluation which would have allowed us to measure any impact and attribute this to the intervention. Where possible, we attempted to measure impact of intervention using crude measures such as comparison of the number of pre- and postintervention transmission events, but it was judged that this method was too crude to obtain valid and publishable results.

\section{Management of cases}

Notified cases were managed by the Greater Manchester Health Protection Team according to national guidelines [16]: where possible [3], confirmed or probable cases of measles were: (i) isolated (while in a healthcare setting), (ii) advised to avoid contact with vulnerable people, and (iii) excluded from work or educational activities, for the duration of their infectious period. Particular attention was given to cases (or susceptible contacts) who were healthcare workers. For them, a longer exclusion period of $\mathbf{2 1}$ days was advised, in line with UK guidelines [3].

For susceptible household contacts of a probable or confirmed case (i.e. contacts with no history of either natural measles infection or having received a measles containing vaccine), exclusion from educational settings (child-minder, nursery, primary school, secondary school, college or university) was recommended for 10 days following onset of rash in the index case. This was based on the assumption that transmission from index case to contact in this continuous exposure scenario would occur four days before appearance of the rash in the index case (the start of the infectious period for measles) and on the standard 14-day average incubation period for measles defined in national guidelines.

\section{Awareness raising in educational and}

healthcare settings

Awareness of measles and the importance of exclusion from educational settings was increased among teaching staff, students and parents by sending 'warn and inform' letters via school management teams. These letters outlined the symptoms of measles, the need to seek medical assessment while being aware of the potential for transmission, measures to avoid onward transmission (including exclusion and voluntary isolation), and the importance of vaccination in primary prevention of measles.

Raising awareness among clinicians of the infectivity of measles and the importance of reducing risk of transmission to other patients by isolating suspected cases in healthcare settings was achieved proactively by sending a measles bulletin to clinicians, hospitals and local authorities, and reactively by notifying the relevant infection control teams about cases in their institution.

When a cluster was identified, a local outbreak control team was formed to manage it. Within the Health Protection Team, a measles strategy group with a nominated lead maintained an overview. Surveillance data were reviewed at least weekly.

\section{Reducing the pool of susceptible individuals}

Local measures

Both proactive and reactive approaches were used to reduce the pool of susceptible individuals. Where probable or confirmed cases were notified, a complete course of MMR vaccine was advised for all under-immunised household contacts.

Educational settings attended by confirmed or probable cases were targeted. The approach used to increase MMR vaccine uptake varied from sending letters to parents encouraging them to take any under-vaccinated children to their GP for MMR vaccination, to specific school-based immunisation sessions. In school-based immunisations sessions, priority was given to immunising children who had received no MMR dose over those who were partially immunised.

Local OCTs decided whether to offer MMR vaccine to children attending affected schools, via GPs or a school-based campaign depending on the level of susceptibility within the setting, resources available to local public health teams, and on whether a previous school-based immunisation session had already taken place in the setting or not.

In some areas, MMR vaccine was proactively offered in schools with a high number of susceptible children, especially where these schools had links to affected schools [17]. The risk of transmission between schools was highlighted early on by social network analysis (Figure 4). Early awareness and understanding of this potential for transmission allowed local teams to target 'feeder' primary schools (primary schools most likely to host younger siblings of cases in the secondary school) as sites for intervention.

Proactive approaches included: supporting local public health teams in areas most affected by the outbreak to raise awareness of the measles outbreak, promoting MMR vaccination, and raising awareness of the importance of immunity to measles among all clinical staff via a regular measles bulletin.

National campaign

In response to multiple outbreaks in different areas of England and a rise in numbers of cases nationally in 2012 and 2013, a national MMR catch-up vaccination campaign [13] was launched in April 2013, with the aim of achieving a $95 \%$ uptake of one dose of MMR vaccine in 10-16 year-olds by September 2013 . 
Nationally-set indicators were used to measure uptake, which included number of invitations sent and number of MMR vaccine doses ordered by primary care.

The national MMR catch-up vaccination campaign was implemented in two phases.

In Greater Manchester, in phase 1, primary care services were asked to actively identify under-immunised 10-16 year-olds using a variety of methods, including checking their own practices' electronic records [18], and to invite them for MMR vaccination using a method of their choice (usually a letter) and immunise them. GPs were remunerated for this service. Underimmunised 10-16 year-old children were defined as 10-16 year-old children who had received one dose or no dose of MMR vaccine. Priority was given to those who had received no dose of MMR vaccine.

In phase 2, the focus in Greater Manchester was on schools with low uptake. School Health Services review immunisation status when the children enter primary or secondary school and when delivering other vaccinations such as human papillomavirus (HPV) vaccine, and at school leaver's booster. Any 10-16 yearolds under-immunised for measles identified in this way were offered a dose of MMR vaccine.

The local impact of the national MMR catch-up vaccination campaign

A total of 496 (98\%) of General Practices in Greater Manchester participated in the catch-up vaccination campaign. Between May 2013 and October 2013, over 10,000 invitation letters were sent out and 947 doses of MMR vaccine were given by Greater Manchester primary care services taking part in the catch-up campaign. Phase 1 of the catch-up campaign ended in September 2013, and no further letters were sent out beyond this point. Further eligible children will have been identified and immunised during phase 2 of the campaign by school nurses. No further information on numbers of $10-16$ year-olds identified or numbers of 10-16 year-olds immunised is currently available.

An interim analysis of the impact of the catch-up campaign nationally has been recently published [19], which suggested achievement of the target of $95 \%$ of 10-16 year-olds having had at least one dose of MMR vaccine. Indirect impacts such as raising awareness of the risks of measles and the benefits of MMR vaccination are likely to have had a positive impact.

\section{Discussion and conclusions}

The descriptive epidemiology of this outbreak was consistent with multiple introductions of measles into partially susceptible communities resulting in a series of discrete outbreaks rather than Greater Manchester wide transmission. Some local authorities had localised outbreaks, often amplified and maintained in educational settings, in particular secondary schools, reflecting known cohorts of low coverage. This was demonstrated by the social network diagrams and the age groups affected. The outbreak control team found social network analysis useful in the early phases of localised outbreaks associated with a particular setting to understand transmission patterns and enable prioritisation of control measures. Other local authorities had only sporadic cases with no known link between them. This observed pattern may be partly the result of artefact, with contributing factors such as underreporting of cases to the health protection team, and partly a reflection of different transmission patterns in different areas, with possible contributing factors including: different MMR vaccine uptake rates, difference in potential for onward transmission (for example, cases who do not attend educational settings), differences in social networks and socialising behaviours. This is in contrast to an earlier large outbreak in Merseyside (40 miles away from Greater Manchester), where the key transmission events were in healthcare settings [20].

Patterns of transmission differed between local authority areas. Two neighbouring areas with similar current MMR vaccine uptake levels experienced different transmission patterns: one had a localised secondary school-focused outbreak which was rapidly controlled with a proactive approach to increasing MMR vaccine uptake in the susceptible population; the other had a more widespread and longer outbreak associated with a variety of educational settings and family clusters, managed reactively. This may be due to the educational systems within the areas (including catchment areas of the schools) or due to the outbreak management strategy.

Northern areas of Greater Manchester reported more cases and outbreaks than southern areas. Although current uptake rates for MMR vaccine are high in many northern areas of Greater Manchester, historical uptake rates were not, and the estimated size of the susceptible population was higher in these areas. Absolute numbers of susceptible individuals rather than current vaccine uptake rates should be taken into account when prioritising areas for proactive control measures.

In this outbreak, the genotype confirmed was D8, a different genotype to the recent large outbreak in the neighbouring area of Merseyside [15], and the same genotype but different strain to the recent large outbreak in South Wales [20]. D8 strain has been identified in other areas: an outbreak in North Wales, cases in Cheshire and a concurrent outbreak in the traveller community [16]. The different genotyping highlighted the limited transmission risks and social networks between the two neighbouring cities.

Investigation of social settings was useful to track transmission and identify targets for intervention particularly in the early phases of the outbreak. The pictorial representation of the contextual settings along with its association with the case provided an 
understanding of the progression of the spread of the disease and helped plan preventive actions.

The sharp decrease in notification of cases in the summer holidays (from the end of July to early September) supports the hypothesis that schools were the sites of transmission during this outbreak.

The public health actions of note included: reducing likelihood of onward transmission by excluding susceptible household contacts of cases from educational settings and reducing the size of the susceptible population by proactive MMR immunisation targeting highrisk educational settings.

Increasing MMR vaccine uptake in target groups via GPs was carried out as part of different aspects of the outbreak response: as part of local outbreak management, on instruction from the local outbreak control team; and as part of the national MMR vaccination catch-up campaign; this was directed nationally and GPs only commenced systematic activity once the campaign was launched in late April 2013.

Exclusion of unimmunised household contacts from educational settings was voluntary and informal. In the majority of cases, parents and schools agreed to this request but compliance with exclusion was not monitored.

The importance of isolation of infectious individuals in controlling the spread of measles has been reported on previously [21], and anecdotal evidence from this outbreak suggests that excluding infectious cases and their susceptible household contacts from educational settings was one of the most effective measures in controlling local outbreaks.

In the response to some local outbreaks, high-risk schools were identified usually based on social/educational networks and levels of MMR vaccine uptake. These schools were then targeted proactively. This seemed to be associated with rapid control in some areas. Uptake in this scenario may be better than proactive campaigns in the absence of an outbreak.

This outbreak was not associated with specific hardto-reach groups [22] but was in the wider population. Such outbreaks highlight the problem of cohorts of under-vaccinated children. If such outbreaks continue to occur, Europe will not achieve its goal of measles elimination by 2015 [23].

This paper describes the epidemiology and management of a prolonged outbreak of measles. Data were collected for case management purposes. Standard operating procedures evolved during the outbreak. Evaluation of individual control measures was not planned before the outbreak with resultant limitations. Children interact both inside and outside of school: it was impossible to separate secondary schools and the associated out-of-school social networks as sites of transmission.

We have described a measles outbreak that predominantly affected an under-immunised cohort of teenagers and young adults, with key transmission and amplification events associated with secondary schools. Of key importance for public health practice were the thorough investigation of outbreaks, developing understanding of local transmission supported by the use of social network analysis, and the multifacetted approach to control measures, with a bundle of public health measures (both reactive and proactive) focussing on the two key elements of improving MMR vaccine uptake in the susceptible population and excluding infectious cases from settings with a high potential for onwards transmission (such as secondary schools).

\section{Acknowledgements}

We gratefully acknowledge all the NHS, PHE (formerly HPA), schools and local authority staff who contributed to outbreak management.

\section{Conflict of interest}

None declared.

Authors' contributions

MP RM DF WW VB KS CK and GM were involved in the management of the outbreak. WW and KS undertook the main data analysis. RWCW analysed the schools data. The overall paper outline was developed by WW RM and MP. All authors drafted sections of the paper. All authors commented on drafts and approved the final version.

\section{References}

1. World Health Organization (WHO) Regional Office for Europe. Guidelines for measles and rubella outbreak investigation and response in the WHO European Region. Copenhagen: WHO. 2013. Available from: http://www.euro.who.int/_data/assets/ pdf_file/o003/217164/OutbreakGuidelines-updated.pdf?ua=1

2. European Centre for Disease Prevention and Control (ECDC). Annual epidemiological report: Reporting on 2011 surveillance data and 2012 epidemic intelligence data. Stockholm: ECDC. 2013. Available from: http://www.ecdc.europa.eu/en/ publications/Publications/annual-epidemiological-report-2013. pdf

3. Health Protection Agency (HPA). HPA National Measles Guidelines. Local \& Regional Services. Version 1.2. London: HPA. 28 Oct 2010. Available from: https://www.gov.uk/ government/uploads/system/uploads/attachment_data/ file/322932/National_Measles_Guidelines.pdf

4. Ramsay M. Measles in England 2012 and 2013. Presentation. London: Public Health England. [Accessed 18 Mar 2013]. Available from: https://www.gov.uk/government/uploads/ system/uploads/attachment data/file/192611/Presentation by_Mary_Ramsay_-_Measles_in_England_2012__2013.pdf

5. Taylor B, Miller E, Farrington CP, Petropoulos MC, FavotMayaud I, Li J, et al. Autism and measles, mumps, and rubella vaccine: no epidemiological evidence for a causal association. Lancet. 1999:353(9169):2026-9. http://dx.doi.org/10.1016/ S0140-6736(99)01239-8 PMID:10376617

6. Farrington CP, Miller E, Taylor B. MMR and autism: further evidence against a causal association. Vaccine. 2001;19(27):3632-5. http://dx.doi.org/10.1016/S0264410X(01)00097-4 PMID:11395196 
7. Health Protection Agency (HPA). Completed primary courses at two years of age: England and Wales, 1966-1977, England only 1978 onwards. London: HPA. [Accessed 9 Dec 2014]. Available from: https://www.gov.uk/government/uploads/system/ uploads/attachment_data/file/356061/The_Immunisation_ Coverage_1966_2012_13.pdf

8. Choi YH, Gay N, Fraser G, Ramsay M. The potential for measles transmission in England. BMC Public Health. 2008;8(1):338. http://dx.doi.org/10.1186/1471-2458-8-338 PMID:18822142

9. Health Protection Agency (HPA). Confirmed measles cases in England and Wales - update to end-June 2012. Health Protection Report. 2012; 6(34). 24 August 2012. Available from: http://webarchive.nationalarchives.gov.uk/20140714084352/ http://www.hpa.org.uk/hpr/archives/2012/news3412.htm

10. The Health and Social Care Information Centre. Screening and Immunisations team. NHS Immunisation Statistics, England 2011-12. HSCIC, 27 November 2012. [Accessed 17 Jun 2013]. Available from: http://www.hpa.org.uk/web/ HPAweb\&HPAwebStandard/HPAweb_C/1195733783627

11. Public Health England (PHE). Calculating MMR coverage: ready reckoner tool 2013. London: PHE. 25 April 2013. Available from: https://www.gov.uk/government/publications/ calculating-mmr-coverage-ready-reckoner-tool-2013

12. Glasswell A, Bishop L. Fraser G for the Evaluation Subgroup of the London Immunisation Steering Group. Evaluation of London Primary Care Trust MMR catch-up programmes in response to call of the chief medical officer: September 2008 - May 2009. April 2010. Available from: www.londonhp.nhs.uk/wp-content/ uploads/2011/03/MMR-Evaluation-Report.doc

13. Public Health England (PHE). National MMR vaccination catchup programme announced in response to increase in measles cases. Press release. London: PHE. 25 April 2013. Available from: https://www.gov.uk/government/news/national-mmrvaccination-catch-up-programme-announced-in-response-toincrease-in-measles-cases

14. Hungerford D, Vivancos R, Cleary P, Welfare W. Country report - United Kingdom: Measles and rubella surveillance in England. ECDC Surveillance Report: Measles and rubella monitoring. Stockholm: ECDC. March 2013. Available from: http://www. ecdc.europa.eu/en/publications/Publications/measles-rubellamonitoring-report-march-2013.pdf

15. Vivancos R, Keenan A, Farmer S, Atkinson J, Coffey E, Dardamissis E, et al. An ongoing large outbreak of measles in Merseyside, England, January to June 2012. Euro Surveill. 2012;17(29):20226. Available from: http://www. eurosurveillance.org/ViewArticle.aspx?Articleld=20226 PMID:22835470

16. Health Protection Agency (HPA). Post exposure prophylaxis for measles: Revised guidelines. London: HPA. May 2009. Available from: http://webarchive.nationalarchives.gov. uk/20140714084352/http://hpa.org.uk/webc/hpawebfile/ hpaweb_c/1238565307587

17. Pegorie M, Munslow G, McCann R, Shankar K, Fiefield D, Philp R. When measles meets the Wakefield cohort: managing a secondary school outbreak -a triumph of partnership and professionalism over the barriers of transition. 5 Nations Health Protection Conference. Dublin. 14-15 May 2013. Available from: http://5nations.org.uk/wp-content/ uploads/2013/01/5-Nations-Health-Protection-ConferenceFinal-Programme.pdf

18. Public Health England (PHE). MMR Action Plan. 15 May 2013. London: PHE. Available from: https://www.gov.uk/government/ uploads/system/uploads/attachment_data/file/206243/PHE MMR_Action_Plan_June_2013.pdf

19. Public Health England. (PHE). Evaluation of vaccine uptake during the 2013 MMR catch-up campaign in England - Report for the national measles oversight group. London: PHE. 2013. Available from: https://www.gov.uk/government/uploads/ system/uploads/attachment_data/file/28589o/Evaluation_of_ the_2013_MMR_catch-up_campaign_in_England.pdf

20. Health Protection Agency (HPA). Measles cases in England: update to end-April 2013. Health Protection Report. 2013;7(23). 7 June 2013. Available from: http://webarchive. nationalarchives.gov.uk/20140714084352/http://www.hpa. org.uk/hpr/archives/2013/news2313.htm

21. Delaporte E, Wyler Lazarevic CA, Iten A, Sudre P. Large measles outbreak in Geneva, Switzerland, January to August 2011: descriptive epidemiology and demonstration of quarantine effectiveness. Euro Surveill. 2013;18(6):20395. PMID:23410259

22. European Centre for Disease Prevention and Control (ECDC). Technical Report. Review of outbreaks and barriers to MMR vaccination coverage among hard-to-reach populations in Europe. Stockholm: ECDC; 2013. Available from: http://ecdc. europa.eu/en/publications/Publications/MMR-vaccinationhard-to-reach-population-review-2013.pdf
23. European Centre for Disease Prevention and Control (ECDC). Special Report. Implementing the ECDC Action Plan for Measles and Rubella. Stockholm: ECDC; 2014. Available from: http:// www.ecdc.europa.eu/en/publications/Publications/measlesrubella-implementing-action-plan.pdf 